\title{
Impactos de la COVID-19 en la violencia contra las mujeres. El caso de Bogotá (Colombia)
}

\author{
Impacts of COVID-19 on violence against women. The case of Bogotá \\ (Colombia)
}

Liliana Chaparro Moreno', Heyder Alfonso²

\section{Resumen}

La literatura reconoce que las condiciones estructurales de vulnerabilidad de las mujeres que se derivan de los roles asociados al cuidado y al trabajo doméstico y de su precarización laboral, entre otros, favorecen el aumento de la violencia en su contra, y esta se exacerba en las condiciones de confinamiento y aislamiento social generadas por la pandemia de la COVID-19. El artículo estudia los mecanismos dispuestos en Bogotá (Colombia) para enfrentar la violencia contra las mujeres y los desafíos que aún se presentan.

Palabras claves: Violencia contra las mujeres, violencia doméstica, COVID-19, coronavirus, Bogotá, Colombia.

\section{Abstract}

The literature recognizes that the structural conditions of women's vulnerability derived from the roles associated with care and domestic work and their job insecurity, among others, allow the violence increase against them, which is exacerbated in confinement conditions and social isolation generated by the Covid-19 pandemic. The article studies the mechanisms developed in Bogotá (Colombia) to face violence against women and the challenges related.

Keywords: Violence against women, domestic violence, Covid-19, coronavirus, Bogotá, Colombia.

\footnotetext{
1. Docente de Ia Maestría en Defensa de Ios Derechos Humanos y del Derecho Internacional Humanitario ante Organismos, Tribunales y Cortes Internacionales, Universidad Santo Tomás.

Correo electrónico: lilianachaparro@usantotomas.edu.co

ORCID: https://orcid.org/0000-0002-2063-0320

2. Docente de la Maestría en Defensa de los Derechos Humanos y del Derecho Internacional Humanitario ante Organismos, Tribunales y Cortes Internacionales, Universidad Santo Tomás.

Correo electrónico: heyderalfonso@usantotomas.edu.co

ORCID: https://orcid.org/0000-0003-2929-3030
}

https://doi.org/10.22490/24629448.4195 
El aumento de la violencia contra las mujeres $(\mathrm{VcM})$ en el marco de la pandemia era predecible (1). Existe evidencia del incremento de dicha violencia en desastres naturales, guerras y crisis humanitarias (2). Las epidemias del zika y ébola sugirieron una transformación de la VcM, pues se escaló su impacto $(3,4,5,6)$ y demostraron que no incorporar en la respuesta las desigualdades de género profundizó aún más esta problemática (5).

En Colombia, la cuarentena se decretó el 24 de marzo de 2020 y desde esa fecha hasta el 22 de junio se registraron $107 \mathrm{fe}-$ minicidios (7). En Bogotá, donde el confinamiento inició el 20 de marzo, se recibieron hasta el 15 de junio 2627 llamadas por VcM (8), en su mayoría por violencia psicológica (figura 1); este dato demuestra un incremento del $187 \%$ en atención por líneas telefónicas y del $774 \%$ en líneas virtuales, respectivamente (9). Respecto al año anterior, los feminicidios en Bogotá aumentaron 8,6\% (10).

Figura 1. Porcentaje de solicitudes de atención según tipo de violencia.

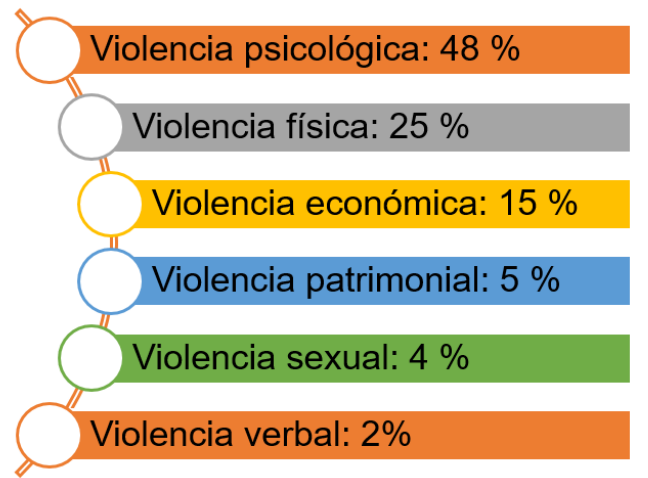

Fuente: Secretaría de la Mujer, Bogotá (8).
La pandemia ha tenido impactos diferenciados para las mujeres y exacerbados debido a la raza, clase y edad $(11,12)$. Según la literatura disponible, la sobrecarga de las labores de cuidado pagas y no pagas, las particulares condiciones que crea el confinamiento - muchas veces en hacinamiento- (2), el aislamiento social, el mayor riesgo al desempleo (12), la sobreexposición a entornos de infección por oficios que implican un contacto mayor con personas contagiadas (12-14) y las limitaciones a la autonomía en la toma de decisiones en salud sexual y reproductiva $(2,11-16)$ han aumentado las afectaciones a las mujeres por la pandemia (2, 12-18) . Asimismo, los impactos de la pandemia para toda la sociedad se han profundizado por las condiciones que enfrentan las mujeres y por las medidas de contención (15).

Los riesgos de género fueron tempranamente alertados por organizaciones sociales (18) y organismos internacionales $(1,19)$, especialmente los ligados con la violencia intrafamiliar y sexual. El confinamiento aumenta el tiempo de contacto con parejas abusivas y el aislamiento social crea menos oportunidades para que agentes externos intervengan y las víctimas busquen ayuda, lo que produce un entorno más dispuesto para la violencia $(2,11,15,20)$. Además, la menor circulación de personas en la calle

1. En Colombia, las cifras recogidas por la Corporación Sisma Mujer establecen que las mujeres dedican a labores de cuidado y trabajo doméstico semanalmente en promedio 50,6 horas, mientras que los hombres destinan 23,9 horas; que la tasa de desempleo de los hombres es de $8,8 \%$ y de las mujeres de $15,4 \%$; que las mujeres representan el $73 \%$ del personal del sector salud y el $86 \%$ de la atención médica residencial, pero reciben el 25 $\%$ menos del salario de los hombres (18). 
posibilita el aumento de la violencia fuera del hogar.

Para el caso de Bogotá, las respuestas a la VcM se han dado en tres niveles: 1) desde normas existentes antes del inicio de la pandemia; 2) desde normas nacionales decretadas luego de la pandemia y; 3) desde normas distritales. Todas ellas, aunque pertinentes, siguen siendo insuficientes para enfrentar un fenómeno que adquiere dimensiones pandémicas.

En relación con las normas ya existentes, la Ley 1257 de 2008 y sus decretos reglamentarios ya presentaban obstáculos antes de la pandemia (21) y se vieron profundizados por el confinamiento. Ejemplo de ello es la dificultad de las mujeres, por las restricciones a la movilidad, para acceder a casas de refugio a fin de lograr protección.

El Gobierno Nacional emitió el Decreto 460 de 2020, en el que se establece la prestación ininterrumpida de las comisarías de Familia con el fin de garantizar la protección de víctimas de violencia intrafamiliar. Aunque novedoso, este decreto desconoce tres aspectos: no todos los lugares del país cuentan con comisarías de Familia, solo se ubican en zonas urbanas y no toda la VcM ocurre al interior de la familia.

En el ámbito distrital se ha considerado como excepción al confinamiento las situaciones de fuerza mayor, incluida la VcM. Aunque las autoridades distritales han favorecido la atención virtual y telefónica, y la solicitud de auxilio a través de mercados y farmacias, es necesario fortalecer los mecanismos de atención presencial.

Las normas existentes aún deben adaptarse a lo que ocurre por el confinamiento y el aislamiento social, so pena de mantener el incremento de feminicidios. Factores que deben mitigarse son los fenómenos como el desistimiento de las denuncias derivada del miedo o de la fase de reconciliación propia del ciclo de la violencia, medidas de protección que no atienden a la gravedad de las amenazas, valoraciones de riesgo que no son efectuadas o lo son de manera tardía, acompańamiento psicosocial que no llega o visitas que deberían practicarse y que el aislamiento retrasa o impide. Podrían crearse presunciones de riesgo que conlleven a una actividad acelerada de las autoridades, como ha ocurrido con otras violencias (22).

Es urgente contemplar la VcM fuera de familia. Muchos de los feminicidios y hechos de violencia ocurren en la calle, el transporte público o a manos de las propias autoridades (18). Mujeres que ejercen el cuidado, la vigilancia y que laboran en el sistema de salud están expuestas a una violencia grave frente a la cual las medidas existentes son aún más limitadas. Abandonar el enfoque familista se convierte en una oportunidad para implementar medidas que protejan a todas las mujeres frente a la violencia y que involucren a los hombres en la solución del problema $(23,24)$. 


\section{Referencias}

1. Comité de expertas MESECVI. Comité de Expertas solicita la incorporación de la perspectiva de género en las medidas que se tomen para la mitigación del COVID-19 y el reforzamiento de acciones para la prevención y atención de la violencia de género [Internet]. 2020. Disponible en: https:// mailchi.mp/dist/comunicado-covid-19-y-el-reforzamiento-de-acciones-para-la-prevencin-y-atencin-de-la-violencia-de-gnero?e $=148 \mathrm{~d} 9 \mathrm{c} 4077 \& \mathrm{fbcli}$ $\mathrm{d}=$ IwAR3Ao4JIlyAXcPktgs5 PqtgHsdaonAsygCyQ9eeKbmps7ufj59nl9d2Nqc4

2. Roesch E, Amin A, Gupta J, García-Moreno C. Violence against women during covid-19 pandemic restrictions. The BMJ. 2020;369. DOI: https://doi. org/10.1136/bmj.m1712

3. United Nations Development Programme. Ebola Recovery in Sierra Leone: Tackling the Rise in Sexual and Gender-based Violence and Teenage Pregnancy during the Ebola Crisis; 2015. Disponible en https://www.undp.org/content/dam/sierraleone/ docs/Ebola\%20Docs./SL\%20FS\%20SGBV.pdf.

4. Oxfam International. Dominican Republic Gender Analysis: A study of the impact of the Zika virus on women, girls, boys and men; 2017.

5. Davies SE, Bennett B. A gendered human rights analysis of Ebola and Zika: locating gender in global health emergencies. International Affairs. 2016;92(5):1041-60. DOI: https://doi. org/10.1111/1468-2346.12704

6. Harman S. Ebola, gender and conspicuously invisible women in global health governance. Third World Quarterly. 3 de marzo de 2016;37(3):524-41. DOI: https://doi.org/10.1080/01436597.2015.1108827

7. Observatorio Feminicidios Colombia. Vivas nos queremos: Dossier de feminicidios en cuarentena. Periodo del 16 de marzo al 16 de junio de 2020 [Internet]. 2020 jun. Disponible en: http://observatoriofeminicidioscolombia.org/attachments/
article/428/Dossier\%20Femincidios\%20Colombia\%20en\%20Cuarentena\%20Junio\%2022.pdf

8. Secretaría Distrital de la Mujer. Reporte atenciones [Internet]. 2020. Disponible en: http://omeg. sdmujer.gov.co/phocadownload/2020/mediciones/ linea $\% 20$ purpura $\% 20$ Reporte $\% 20$ Atenciones.pdf

9. Secretaría Distrital de la Mujer. A dos meses de confinamiento, la Secretaría Distrital de la Mujer sigue acompañándote en casa de diversas maneras [Internet]. 2020. Disponible en: http://omeg.sdmujer.gov. co/index.php/articulos/244-a-dos-meses-de-confinamiento-la-secretaria-distrital-de-la-mujer-sigue-acompanandote-en-casa-de-diversas-maneras

10. Secretaría Distrital de la Mujer. Comunicado de prensa n. 016 [Internet]. 2020. Disponible en: http://www.sdmujer.gov.co/noticias/casos-identificados-alto-riesgo-feminicidio-tendrán-seguimiento-semanal-desde-distrito

11. Viveiros N, Bonomi AE. Novel Coronavirus (COVID-19): Violence, Reproductive Rights and Related Health Risks for Women, Opportunities for Practice Innovation. J Fam Violence. 2020. DOI: https://doi.org/10.1007/s10896-020-00169-x

12. Lokot M, Avakyan Y. Intersectionality as a lens to the COVID-19 pandemic: implications for sexual and reproductive health in development and humanitarian contexts. Sex Reprod Health Matters. 2020;28(1). DOI: https://doi.org/10.1080/264103 97.2020 .1764748

13. Bahn K, Cohen J, Rodgers Y. A feminist perspective on COVID-19 and the value of care work globally. Gend Work Organ. 2020. DOI: https://doi. org/10.1111/gwao.12459

14. Wenham C, Smith J, Morgan R. COVID-19: the gendered impacts of the outbreak. Lancet. 2020;395(10227):846-8.

15. Gausman J, Langer A. Sex and Gender Disparities in the COVID-19 Pandemic. J Womens Health. 2020;29(4):465-6. 
16. Lokot M, Avakyan Y. Intersectionality as a lens to the COVID-19 pandemic: implications for sexual and reproductive health in development and humanitarian contexts. Sex Reprod Health Matters [Internet]. 2020;28(1). DOI: https://doi.org/10.1080 /26410397.2020.1764748

17. del Río Lozano M, García Calvente MDM, Grupo de alumnado del Diploma de Especialización en Género y Salud de la Escuela Andaluza de Salud Pública-Universidad de Granada. Caregiving and the COVID-19 pandemic from a gender perspective. Gaceta Sanit. 2020.

18. Corporación Sisma Mujer. Comportamiento de las violencias contra las mujeres en el marco de la pandemia del Covid-19 en Colombia [Boletín n. ${ }^{\circ} 20$ ]; 2020 .

19. Comisión Interamericana de Derechos Humanos. La CIDH hace un llamado a los Estados a incorporar la perspectiva de género en la respuesta a la pandemia del COVID-19 y a combatir la violencia sexual e intrafamiliar en este contexto [Internet]. 2020. Disponible en: http://www.oas.org/es/cidh/ prensa/comunicados/2020/074.asp

20. Mazza M, Marano G, Lai C, Janiri L, Sani G. Danger in danger: Interpersonal violence during COVID-19 quarantine. Psychiatry Research. 2020;289:113046.

21. Red Nacional de Mujeres. Análisis de la Ley 1257 de 2008 en sus diez años de implementación; 2018.

22. Corte Constitucional. Auto 009. 2015.

23. Betron M, Gottert A, Pulerwitz J, Shattuck D, Stevanovic-Fenn N. Men and COVID-19: Adding a gender lens. Glob Public Health. 2020;15(7):10902.

24. Wenham C, Smith J, Morgan R. Covid-19 is an opportunity for gender equality within the workplace and at home Could covid-19 help unravel gender norms? BMJ-British Medical Journal. 2020;369:m1546. doi: https://doi.org/10.1136/ bmj.m 1546 